Hydrology and Earth System Sciences, 10, 93-100, 2006

www.copernicus.org/EGU/hess/hess/10/93/

SRef-ID: 1607-7938/hess/2006-10-93

European Geosciences Union

\title{
Experimental and theoretical memory diffusion of water in sand
}

\author{
G. Iaffaldano ${ }^{1, *}$, M. Caputo ${ }^{1,2}$, and S. Martino ${ }^{3}$ \\ ${ }^{1}$ Department of Physics, University "La Sapienza", Rome, Italy \\ ${ }^{2}$ Department of Geology and Geophysics, Texas A\&M University, College Station, Texas \\ ${ }^{3}$ Department of Earth Sciences, University "La Sapienza", Rome, Italy \\ *now at: Geophysics Section, Ludwig-Maximilians University, Munich, Germany
}

Received: 18 May 2005 - Published in Hydrology and Earth System Sciences Discussions: 2 August 2005

Revised: 25 October 2005 - Accepted: 29 December 2005 - Published: 15 February 2006

\begin{abstract}
The basic equations used to study the fluid diffusion in porous media have been set by Fick and Darcy in the mid of the XIXth century but some data on the flow of fluids in rocks exhibit properties which may not be interpreted with the classical theory of propagation of pressure and fluids in porous media (Bell and Nur, 1978; Roeloffs, 1988).

Concerning the fluids and the flow, some fluids carry solid particles which may obstruct some of the pores diminishing their size or even closing them, some others may chemically and physically react with the medium enlarging the pores; so permeability changes during time and the flow occurs as if the medium had a memory.

In this paper we show with experimental data that the permeability of sand layers may decrease due to rearrangement of the grains and consequent compaction, as already shown qualitatively by Elias and Hajash (1992). We also provide a memory model for diffusion of fluids in porous media which fits well the flux rate observed in five laboratory experiments of diffusion of water in sand. Finally we show that the flux rate variations observed during the experiments are compatible with the compaction of sand, due to the amount of fluid which went through the grains locally, and therefore with the reduction of porosity.
\end{abstract}

\section{Introduction}

Darcy's law, which states that the flux is proportional to the pressure gradient, has led many authors to set rigorous equations representing the interaction between the porous media and the fluid flow (Bear, 1972; Sposito, 1980; Steefel and Lasaga, 1994; Dewers and Ortoleva, 1994; Indelman and Abramovici, 1994; Cushman and Moroni, 2001). In spite of this, some data exhibit properties which may not be in-

Correspondence to: G. Iaffaldano

(giampiero@geophysik.uni-muenchen.de) terpreted neither with the classical theory of propagation of pressure and fluids in porous media (Bell and Nur, 1978; Roeloffs, 1988) nor adequately with many of the new theories. Permeability changes may occur in time if the fluid carries solid particles which obstruct some of the pores or chemically reacts with the medium enlarging them. If that is the case, then the flow would occur as if the medium had a memory, meaning that at any instant the process of diffusion is also affected by the history of pressure and flow. These phenomena must be taken into account when modeling diffusion of fluids in porous media.

The scope of this paper is to show quantitatively that the permeability of sand layers decreases due to the rearrangement of the grains and consequent mechanical compaction (Elias and Hajash, 1992). By rewriting the constitutive equation of diffusion with memory formalism, we provide a new model for diffusion of fluids in porous media capable to describe permeability changes observed in the flux rate through the sand samples.

\section{Laboratory experiments}

The experiments were designed to obtain flow measurements through a porous layer with constant hydraulic pressure difference between the boundary surfaces.

The selected medium is sand, which showed an adequate compaction and therefore considerable permeability and flux rate variations over time. The grain size distribution is shown in Fig. 1. The percentiles are shown on top of histogram and the weighted mean value of the grain size is $\langle s\rangle=0.27 \mathrm{~mm}$. Sand density was estimated to be $\rho_{S}=(2.4 \pm 0.1) \mathrm{g} \cdot \mathrm{cm}^{-3}$ for all the experiments.

We used water as fluid, its temperature during all experiments was $(19 \pm 1)^{\circ} \mathrm{C}$.

Figure 2 is a sketch of the instrument assembled for the diffusion experiments.

(C) 2006 Author(s). This work is licensed under a Creative Commons License. 


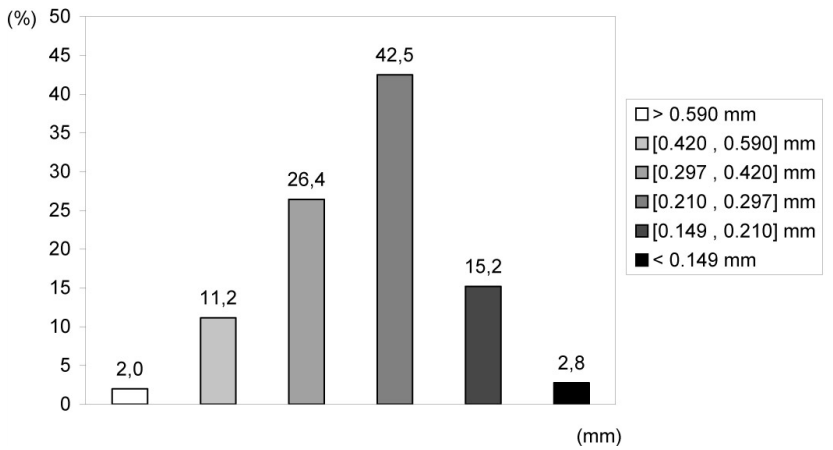

Fig. 1. Grain size distribution for the sand used in the experiments. Weighted mean value of the grain size is $0.27 \mathrm{~mm}$.

Water-saturated sand is kept in the cell for medium, a cylinder shaped metal box of height $l=(11.6 \pm 0.1) \mathrm{cm}$ and surface's inner diameter $D_{I}=(10.1 \pm 0.1) \mathrm{cm}$. The condition of saturation was obtained slowly filling the empty cell with dry sand and water alternately and removing the surplus water. The cell was closed once completely filled. This ensures that the water pressure inside the cell is the atmospheric pressure. The next step is to obtain the value for the initial pressure of the water pressure inside the cell. In Fig. $2 R, R_{I}$ and $R_{U}$ are water-taps and $R$ is also water source; $T$ is a tank with input gate $I$ and output gate $U ; H=(212 \pm 1) \mathrm{cm}$.

The water-taps $R$ and $R_{I}$ are initially switched on while $R_{U}$ is off; this way the pressure inside the cell increases and, after some time has passed, reaches the value of atmospheric pressure plus the pressure due to the $H$ - height water column. Once the pressure is the same through the medium, the apparatus is ready.

Opening $R_{U}$ the pressure on the boundary plane in $x=l$ is equal to the pressure of a water column of height $H$ plus the atmospheric one, while the pressure on the boundary plane in $x=0$ is the atmospheric pressure and so water starts to flow through porous medium and runs out from $R_{U}$. Note that the column is always of height $H$ because the surplus water from the tap flows out from the gate $U$. This way a constant pressure difference is maintained between the boundary planes in $x=l$ and $x=0$, which was verified during all the experiments using pressure gauge $B$.

Measurements of water flow at the boundary surface in $x=0$ were obtained by storing water in a small container with capacity of about $100 \mathrm{~cm}^{3}$ and taking note of the relative time interval with $10^{-2} \mathrm{~s}$ precision chronometer. However, the main error on time is the one due to the experimenter who starts and stops the chronometer, as explained below.

The water mass in the container was measured using a $10^{-4} \mathrm{~g}$ precision scale.

In order to diminish the error of the experimenter and of the devices the water mass in each container was measured three times with the scale, experimenter error in starting and stopping chronometer was evaluated to be $2 \cdot 10^{-1} \mathrm{~s}$ and each

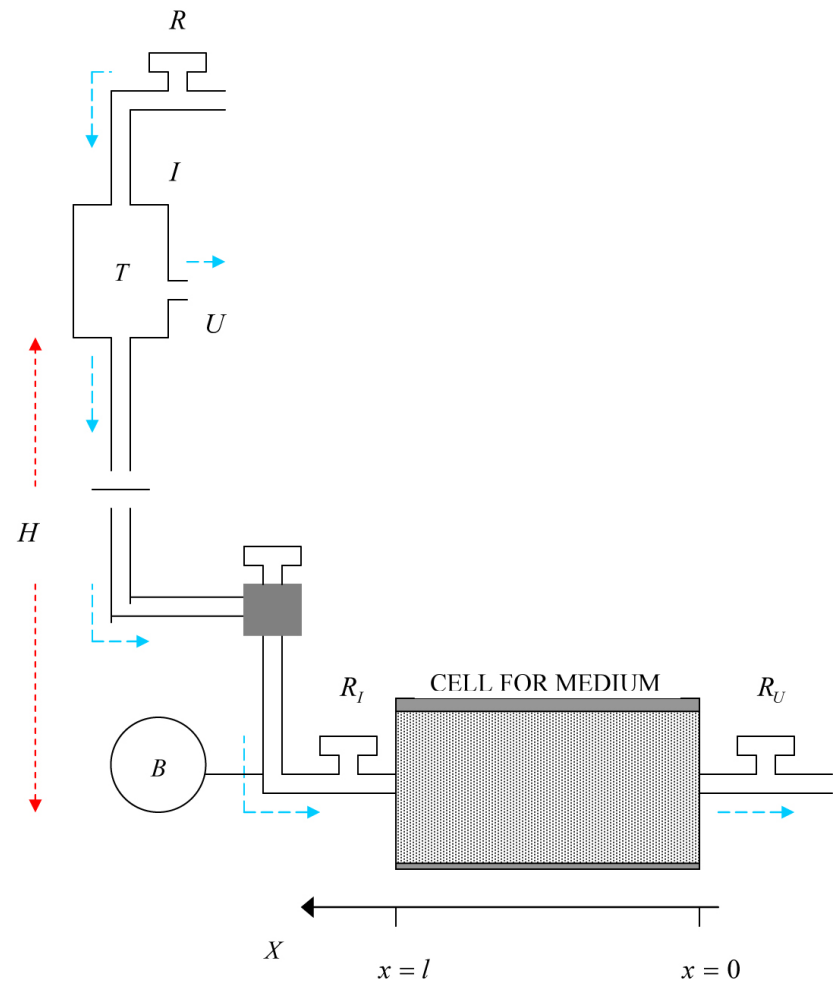

Fig. 2. Experimental device used in this study. A water column of height equal $\mathrm{H}$ generates a pressure on one side of the porous medium. Flux measures are token on the other side of the medium.

flow measure is the average of three containers filled in rapid succession. The estimated relative error in the flux is then about $2 \%$.

All the experiments were managed the same way mentioned above, with the same value of $H$ and with five different samples of the same type of sand. The following figures (Figs. 3-7) show all experimental data collected. Collection is limited to about $10 \mathrm{~h}$, when the fluid flows very slowly, steady rate has been reached and compaction is over. The solid line in each figure is the theoretical flux that best fits the experimental data. The memory formalisms leading to the theoretical flux is introduced in the following.

Note that for each experiment in the first few hours the flux rate steadily decreases defining a transient phase. It appears that in several hours, seemingly less than 10 , the flux establishes to a value that is about $70 \%$ of the initial one (only in experiment 5 it is about $46 \%$ of initial value). The Reynolds number $\left(R_{e}=\rho_{F} V D \mu^{-1}\right.$, where $\rho_{F}$ is fluid density, $D$ is the grain size and $V$ is the volume of flowing water per unit of time and surface) ensures whether the flow is laminar or turbulent. Assuming $D=\langle s\rangle$ and $V \cong 0.81 \mathrm{~cm} \cdot \mathrm{s}^{-1}$ (which was computed in the worst of cases assuming $\left.q \cong 65 \mathrm{~g} \cdot \mathrm{s}^{-1}\right)$, it results $R_{e}=2.2$ which is less than 10 , commonly accepted as threshold for the turbulent motion. 


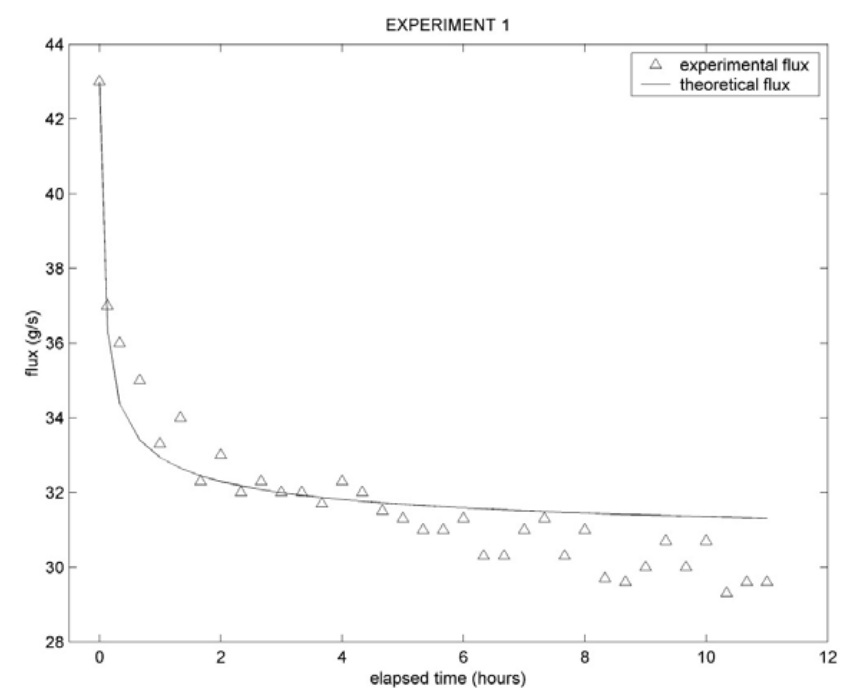

Fig. 3. Flux results (triangules) from experiment number one. An initial transient of about 3 to $4 \mathrm{~h}$ is visible, afterwards the flux reaches a steady state. Overimposed solid line rapresents the theoretical curve which best fits the experimental data.

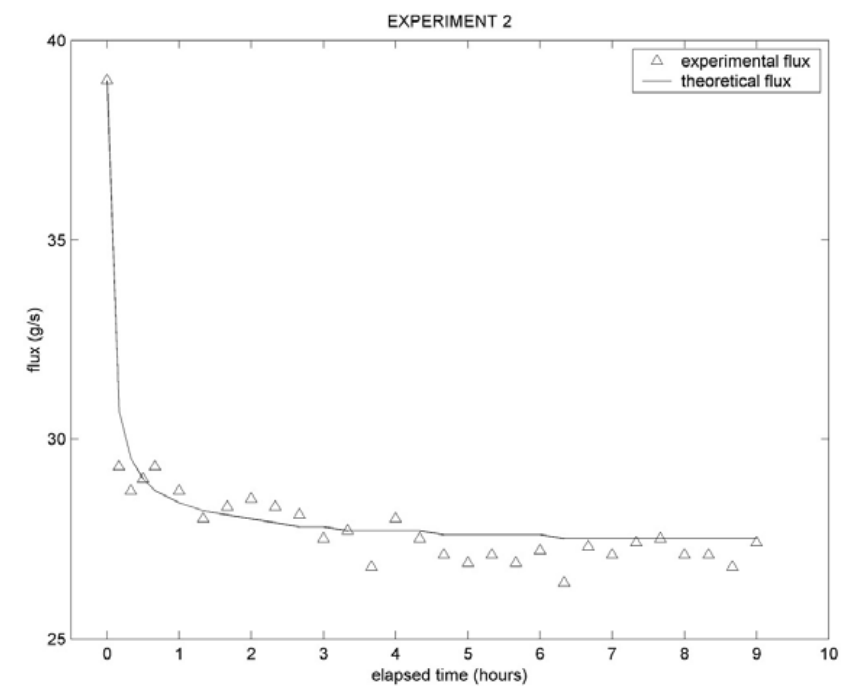

Fig. 4. Same as Fig. 3, for the experiment number two.

Opening the cell for medium after each experiment we observed a height reduction of the sand of about $3-4 \mathrm{~mm}$, that is about $3 \%$ of the porous media volume, and this is an evidence of mechanical compaction.

In order to account for the variation of flux rate in terms of porous media volume reduction we used empirical Fair and Hatch law (1933) for permeability $k$ (Bear, 1972)

$k=C_{M} z^{3} /(1-z)^{2}$

where $z$ is medium porosity and $C_{M}$ is a geometrical medium dependent coefficient introduced to account for the grain

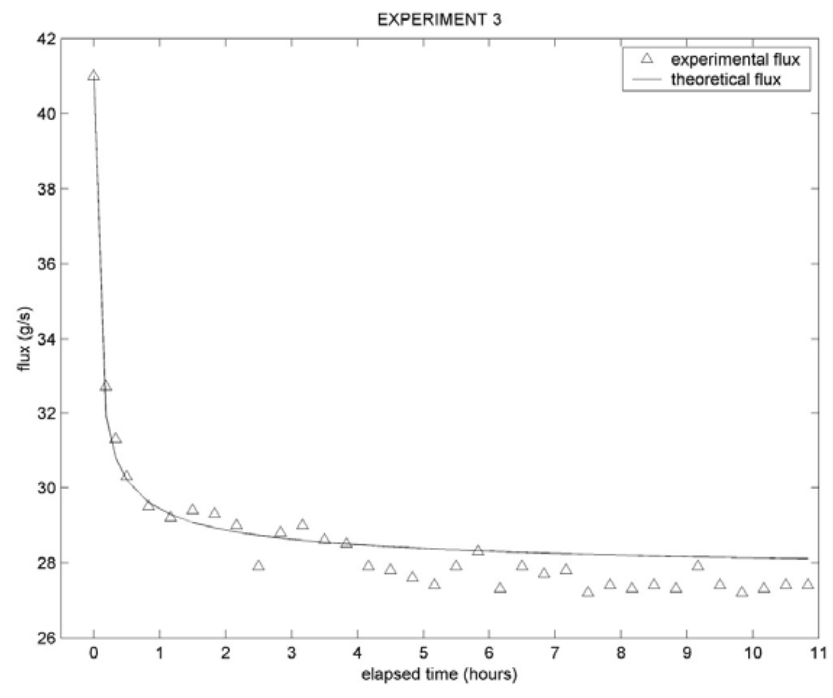

Fig. 5. Same as Fig. 3, for the experiment number three.

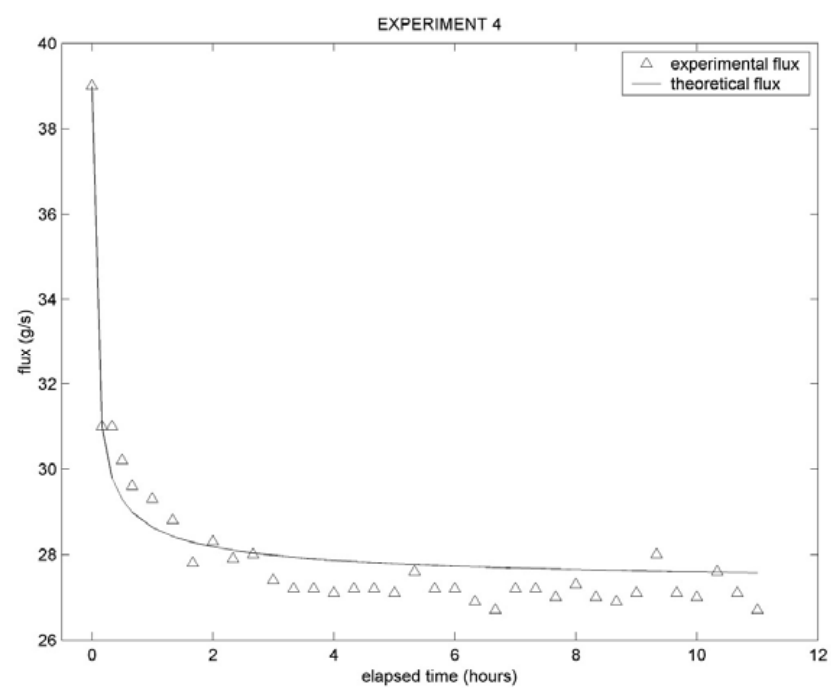

Fig. 6. Same as Fig. 3, for the experiment number four.

size distribution, grains shape and chemical properties of the medium.

The mass of sand in the cell in each experiment was $m=(1550 \pm 30) \mathrm{g}$ (dry sand) and no sand went out from cell during the experiment. Using the Fair and Hatch law with $\rho_{S}=(2.4 \pm 0.1) \mathrm{g} \cdot \mathrm{cm}^{-3}$, it results that $\Delta k \%=(26 \pm 3) \%$. This permeability reduction justifies experimentally observed flux rate reduction.

\section{Modeling the flux variations}

Our experiments show how the diffusion process departs from the Darcy's law. Compaction occurs during time and diffusion is affected by the previous amount of fluid which 


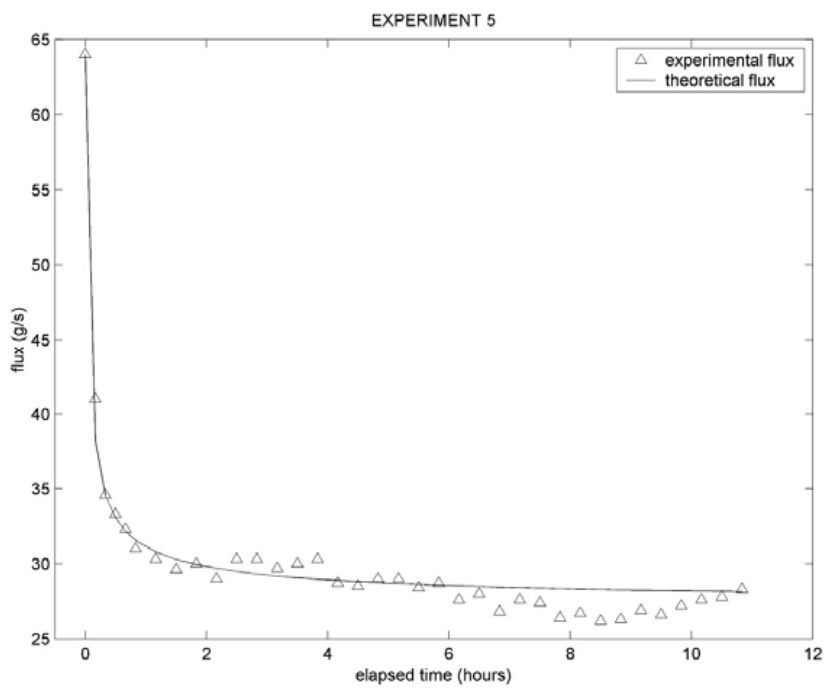

Fig. 7. Same as Fig. 3, for the experiment number five.

went through the pores, so that a new formalism is required. We modified as follows the original Darcy's law

$\bar{q}(\bar{x}, t)=-c \bar{\nabla} p(\bar{x}, t)$

where $p$ is fluid pressure in the porous medium and $q$ is fluid flow through medium, introducing a derivative of fractional order $n$ (Caputo, 2000):

$\gamma \bar{q}(\bar{x}, t)=-\left[c+d \frac{\partial^{n}}{\partial t^{n}}\right] \bar{\nabla} p(\bar{x}, t)$

$a p(\bar{x}, t)=\alpha \rho(\bar{x}, t)$

$\operatorname{div} \bar{q}(\bar{x}, t)+\frac{\partial \rho(\bar{x}, t)}{\partial t}=0$

where $\rho$ is variation of fluid density in medium from the undisturbed condition and $\gamma, c$ and $d$ are real numbers modulating memory formalism, $\alpha / a$ is the bulk modulus of the fluid. In Eq. (3) the flux can be seen as a linear combination of $\bar{\nabla} p(\bar{x}, t)$ and its fractional order derivative; the parameters $c / \gamma$ and $d / \gamma$ are the coefficient of the combination. The definition of fractional order derivative is (Caputo, 1967; Podlubny, 1999 )

$f^{(n)}(t)=\frac{\partial^{n} f(t)}{\partial t^{n}}=\frac{1}{\Gamma(1-n)} \int_{0}^{t} \frac{f^{(1)}(u)}{(t-u)^{n}} d u$

where $n \in[0,1[$ and $\Gamma$ is the Gamma function. Essentially the derivative of fractional order $f^{(n)}(t)$ is a weighted mean of the first order derivative $f^{(1)}(u)$ in the time interval $[0, t]$, which is a sort of feedback mechanism. That is, the values of $f^{(1)}(u)$ at time $u$ far apart from $t$ are given smaller weight than those at time $u$ closer to $t$. Hence, the weights are increasingly smaller with increasing time separation from $t$ to

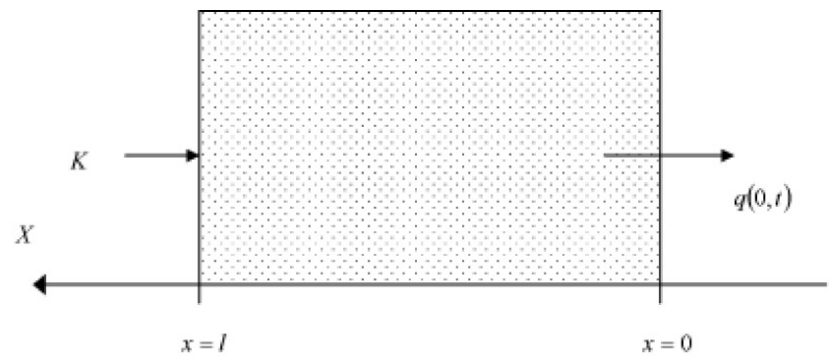

Fig. 8. Sketch of the porous slab with its assumed reference frame.

imply that the effect of past is fading with increasing time. When $n=0$ and $f(0)=0$ the fractional derivative reduce to the functions themselves.

The introduction of fractional derivatives in the constitutive equations of the phenomena studied in geophysics is not new. They have been already used to model the rheological properties of solids (Bagley and Torvik, 1986; Le Mehaute and Crépy, 1983), to model the frequency independent quality factor (Caputo, 1967), to successfully model the fennoscandinian uplift (Körnig and Müller, 1989), to show that the constitutive equation of polarizable media, in the time domain, is represented by a relation containing these derivatives (Caputo and Plastino, 1998). The derivatives of fractional order were also succesfully used in other fields of research like electromagnetism (Jacquelin, 1984), biology (Caputo, 2002b; Cesarone, 2002), chaos (Mainardi, 1996) and economy (Caputo and Kolari, 2001; Caputo, 2002a).

The equations resulting from our procedure are phenomenological. However the reputation of this type of equations, as stated in recent motivations for assigning Nobel prizes for physics, has been rehabilitated for their important contribution given in various forms to the rapid developments of the superconductive materials.

These phenomenological equations, when adequately verified with experimental data, represent a step forward in respect to the usual empirical equations which are still very useful in many branches of applied science and technology.

It is noteworthy to observe how the memory functions capture the past. What the fractional derivative memory functions are remembering is their past values as defined by Eq. (9), which implies that the function is constructed by adding to the initial value the successive weighted increments over time. The increments per unit time are represented by the first order derivative under the integral sign and the weights are represented by the factor of the first order derivative in Eq. (6), which are decreasing with increasing time separation from $t$. Thus, a variable's value is a weighted mean of its past value.

In order to fit our experimental data with memory model we find the Green function of the flux $q(0, t)$ when diffusion occurs through a slab of thickness $l$ with pressure boundary conditions, neglecting the atmospheric pressure which is a 
Table 1. Fitting parameters with their errors for each experiment.

\begin{tabular}{lcccc}
\hline & $n$ & $d / \gamma\left(s^{1+n}\right)$ & $A D\left(g \cdot s^{-1}\right)$ & $q_{A S}\left(g \cdot s^{-1}\right)$ \\
\hline experiment 1 & $0.46 \pm 0.01$ & $0.008 \pm 0.001$ & 0.8 & 30.3 \\
experiment 2 & $0.58 \pm 0.01$ & $0.014 \pm 0.002$ & 0.41 & 27.1 \\
experiment 3 & $0.54 \pm 0.01$ & $0.012 \pm 0.002$ & 0.52 & 27.5 \\
experiment 4 & $0.54 \pm 0.01$ & $0.010 \pm 0.001$ & 0.55 & 27.2 \\
experiment 5 & $0.58 \pm 0.02$ & $0.046 \pm 0.003$ & 0.8 & 27.1 \\
\hline
\end{tabular}

common offset (Appendix A),

$p(0, t)=0$

$p(l, t)=K=\mathrm{constant}$

and initial pressure condition

$p(x, 0)=K=$ constant

In order to obtain the flux $q(0, t)$ we solve the Eqs. (3-5) in the Laplace Transform (LT) domain obtaining

$P(x, s)=\frac{K}{s}\left[\frac{e^{B s^{v}(x-l)}-e^{B s^{v}(l-x)}}{e^{B s^{v} l}-e^{-B s^{v} l}}+1\right]$

and

$Q(0, s)=-\frac{d K B}{\gamma s^{\nu}}\left[\frac{1+e^{2 B s^{v} l}}{e^{2 B s^{v} l}-1}\right]$

where

$B=[a \gamma / \alpha d]^{\frac{1}{2}}, v=(1-n) / 2$

and $\mathrm{s}$ is the LT variable.

The $L T^{-1}$ of Eq. (11) is found in the Appendix B and the following expression of boundary flux is obtained

$q(0, t)=-\frac{d B K}{2 \pi \gamma} \int_{0}^{+\infty} \frac{e^{-r t}}{r^{v}}$

$\cdot \frac{2 \sin (\pi \nu)\left[e^{2 M r^{\nu}}-1\right]+4 \sin \left(N r^{\nu}\right) \cos (\pi \nu) e^{M r^{\nu}}}{e^{2 M r^{\nu}}+1-2 \cos \left(N r^{\nu}\right) e^{M r^{\nu}}} d r$

with

$r=$ modulus of $s$

$M=2 B l \cos (\pi \nu)$

$N=2 B l \sin (\pi \nu)$

Note that in Eq. (7) $a / \alpha=\rho_{F} z / k_{B}$, where and $k_{B}$ is bulk modulus of fluid; water values are $\rho_{F}=1 \mathrm{~g} \cdot \mathrm{cm}^{-3}$ and $k_{B}=2.08 \cdot 10^{10} \mathrm{~g} \cdot \mathrm{cm}^{-1} \cdot \mathrm{s}^{-2}$ (Domenico and Schwartz, 1997). Therefore $B=\left[\gamma \rho_{F} z / d k_{B}\right]^{\frac{1}{2}}$ and, assuming for sand $z=0.35$ (Bear, 1972), the boundary flux theoretical solution $q(0, t)$ depends on memory parameter $d / \gamma$ and the order of fractional derivative $n$ through $\nu=(1-n) / 2$.

With extreme values theorem it is seen that

$\lim _{s \rightarrow 0} s Q(0, s)=\lim _{t \rightarrow+\infty} q(0, t)=0$

$\lim _{s \rightarrow \infty} s Q(0, s)=\lim _{t \rightarrow 0} q(0, t)=-\infty$

\section{Fitting the model to experimental data}

The experimental data show that in several hours flux seems close to stabilization. Since we will describe only the transient phase of diffusion $(c=0)$, we obtained the data to fit by subtracting the average of the last few hours of flux (let's call it $\left.q_{A S}\right)$ to the original data. The data of the five experiments indicate that $q_{A S}$ is a good candidate for asymptotic flux and reprocessed data are good to represent the diffusion transient phase that we want to describe.

In order to best fit the memory model to experimental data we minimized the following two variables function

$A D\left(v, \frac{d}{\gamma}\right)=\frac{1}{N_{D}} \sum_{i=1}^{N_{D}}\left|E D_{i}-q\left(t_{i}, v, \frac{d}{\gamma}\right)-q_{A S}\right|$

where $N_{D}$ is the number of experimental data for each experiment, $E D_{i}$ are the data obtained in the laboratory at the time $t_{i}$.

The results of fitting for the five experiments are shown in Table 1.

Note that experiment 5 is a bit different from the others, in fact the initial flux is higher and the transient reaches steady state at about $46 \%$ of the initial value while the others reach steady state at about $71 \%$ of the initial value. It differs from the others most of all in the first hour, after which it is similar to the other in the stationary part. This may be due to a difference in the preparation of the sample which caused a particular distribution of the grains which favoured a preferential path for the water caused an initial flux anomalously high but the asymptotic value of flux is similar to that of the other experiments.

Taking into account only the first four experiments it results that both for $n$ and $d / \gamma$ the average quadratic 
Table 2. Mean values (AV) and average quadratic discrepancy (AQD) for the first four experiments.

\begin{tabular}{ccc}
\hline & $n$ & $d / \gamma\left(s^{1+n}\right)$ \\
\hline AV & 0.53 & 0.011 \\
AQD & 0.04 & 0.002 \\
\hline
\end{tabular}

discrepancy (AQD) is compatible with the relative average value (AV); values are shown in Table 2.

\section{Conclusions}

In the experiments we have observed that flux decreases over time to about $70 \%$ of initial value and that the volume of sand decreases by about 3\%. Using empirical Fair and Hatch law for permeability, the sand volume and flux reductions seem compatible; which proves that mechanical compaction occurring during diffusion is caused by the permeability changes which in turn cause the flux variations.

The classic theory in the case of constant diffusivity with constant boundary and initial conditions, would give a constant flux, contrary to the results of our laboratory experiments.

We rewrote the constitutive equations of the diffusion process introducing a new memory formalism, based on fractional order derivatives. This formalism accounts for the past history of the process.

We successfully fit the new theoretical flux with our experimental data. Note that for each experiment the value of the minimum $A D$ (see Eq. 16) numerically computed is about $2 \%$ of average observed flux and that the order of the fractional derivatives has a standard deviation of $9 \%$ of the average value which. This is rather satisfactory result which confirms the validity of the model.

With the boundary and initial conditions used, we observed that the relaxation time of the flux is about $10 \mathrm{~h}$. This implies that the compaction of the sand in the sample has the same relaxation time. However in terms of memory model, the flux and the associated relaxation time are now defined by two parameters and not only one as in the classic theory. Those parameters are the order of fractional derivative $n$ and $d \mu / \rho_{F} \gamma$, with $\mu$ being the fluid viscosity.

\section{Appendix A}

It is useful to rewrite memory relations in one dimension:

$\gamma q(x, t)=-\left[c+d \frac{\partial^{n}}{\partial t^{n}}\right] \frac{\partial p(x, t)}{\partial x}$

$a p(x, t)=\alpha \rho(x, t)$ $\frac{\partial q(x, t)}{\partial x}+\frac{\partial \rho(x, t)}{\partial t}=0$

In this appendix we find the $L T$ of the Green function of the flux resulting from Eqs. (A1-A3) with boundary and initial condition given by Eqs. (7-9). Computing the $L T$, differentiating with respect to $x$ and then substituting it results that

$\frac{\left(c+d s^{n}\right)}{\gamma} P_{x x}=\frac{1}{\alpha}[a s P-\alpha \rho(x, 0)]$

Here, in order to reduce the number of free parameters and to simplify the formulae, we set $c=0$ which is justified as follow: it seems that in several hours, seemingly less than $10 \mathrm{~h}$, the flux stabilizes but we cannot rule out that it is asymptotically nil. If the flux were constant after $10 \mathrm{~h}$ then the rigorous solution requires that $c \neq 0$, which implies that asymptotically the flux is constant as required by Darcy's law which does not apply here. We have two options:

1. consider the transient phase which is asymptotically nil

2. consider that after the transient phase the flux stabilizes

However, since we have no indication of the asymptotic value, also for simplicity of computation, we studied only the transient phase and set $c=0$.

So Eq. (A4) becomes

$\frac{d}{\gamma} s^{n} P_{x x}=\frac{a}{\alpha} s P-\rho(x, 0)$

which, by substituting $L T$ of pressure equations, can be written as

$P_{x x}=\frac{\gamma a}{\alpha d}\left[s^{1-n} P-s^{-n} p(x, 0)\right]$

The general solution of Eq. (A6) is

$P(x, s)=C_{1}(s) e^{B s^{v} x}+C_{2}(s) e^{-B s^{v} x}+\frac{K}{s}$

where $B=[a \gamma / d \alpha]^{\frac{1}{2}}$ and $\nu=(1-n) / 2$.

With boundary conditions (7) and (8) in Eq. (A7) we obtain the general solution

$P(x, s)=\frac{K}{s}\left[\frac{e^{B s^{\nu}(x-l)}-e^{B s^{\nu}(l-x)}}{e^{B s^{\nu} l}-e^{-B s^{\nu} l}}+1\right]$

Differentiating Eq. (A8) with respect to $x$ and substituting in the $L T$ of Eq. (A3) we obtain

$Q(x, s)=-\frac{d K B}{\gamma s^{v}}\left[\frac{e^{B s^{\nu}(x-l)}+e^{B s^{v}(l-x)}}{e^{B s^{\nu} l}-e^{-B s^{v} l}}\right]$ 


\section{Appendix B}

In this appendix we find the $L T^{-1}$ of Eq. (11), to be fit to experimental data, by integrating $e^{s t} Q(0, s)$ along the path of Fig. B1 below.

When the radius $R_{1}$ of the inner circle $\Gamma_{1}$ goes to infinity and the radius $R_{2}$ of the outer circle $\Gamma_{2}$ goes to zero the residual theorem (RT) states that the integral is equal to the sum of residuals inside the path.

Path of integration in Fig. B1 can be divided as follow

$\Gamma_{T}=\Gamma_{1}+\Gamma_{F H}+\Gamma_{H A}+\Gamma_{A B}+\Gamma_{B D}+\Gamma_{D E}$

and when $R_{1} \rightarrow 0$ we find that $\Gamma_{B D} \rightarrow \Gamma_{C D}$ and $\Gamma_{H A} \rightarrow \Gamma_{H K}$.

Concerning the integral along $\Gamma_{1}$, when the radius $R_{1}$ goes to zero it can be shown by the Taylor development of $Q(0, s)$ near $s=0$ that

$$
\lim _{s \rightarrow 0} s Q(0, s)=\lim _{R_{1} \rightarrow 0}-\frac{2 d K B}{\gamma} \frac{s^{1-v}}{\left(2 B l s^{v}+o\left(s^{2 v}\right)\right)}=0
$$

and so integral along $\Gamma_{1}$ is zero.

To compute integrals along $\Gamma_{C D}$ and $\Gamma_{H K}$, it is useful to rewrite $e^{s t} Q(0, s)$ with $s=R_{2} e^{i \vartheta}$. When $R_{2}$ goes to infinity imaginary exponential can be neglected because limited in $[-1 ; 1], \cos (\vartheta v) \in] 0,1]$ because $\vartheta \in] \pi / 2, \pi] \cup$ ]$-\pi,-\pi / 2]$ and $\nu=(1-n) / 2 \in] 0,1 / 2]$. So the integrals along $\Gamma_{C D}$ and $\Gamma_{H K}$ are nil because function inside integral is nil.

Function $e^{s t} Q(0, s)$ has no singularity in the complex plain except in the origin, already analyzed. For RT, renaming $e^{s t} Q(0, s)=I(s)$, we have that

$$
\begin{aligned}
& \lim _{\substack{R_{1} \rightarrow 0 \\
R_{2} \rightarrow+\infty}}\left[\int_{\Gamma_{T}} I(s) d s\right]=\lim _{\substack{R_{1} \rightarrow 0 \\
R_{2} \rightarrow+\infty}}\left[\int_{-i R_{2}}^{+i R_{2}} I(s) d s\right. \\
& \left.+\int_{\Gamma_{D E}} I(s) d s+\int_{\Gamma_{F H}} I(s) d s\right]=0
\end{aligned}
$$

and so

$$
T L^{-1}[Q(0, s)]=\lim _{\substack{R_{1} \rightarrow 0 \\ R_{2} \rightarrow+\infty}} \frac{1}{2 \pi i}\left[-\int_{\Gamma_{D E}} I(s) d s-\int_{\Gamma_{F H}} I(s) d s\right]
$$

For the integral along $\Gamma_{D E}$ we set $s=r e^{i \pi}$ and obtain

$$
I\left(r e^{i \pi}\right)=I_{D E}(r)=-\frac{d B K}{\gamma} \frac{e^{-r t}}{e^{i \pi v} r^{\nu}} \frac{\left(1+e^{Z r^{v}}\right)}{\left(e^{Z r^{\nu}}-1\right)}
$$

with

$Z=M+i N$

$M=2 B l \cos (\pi v)$

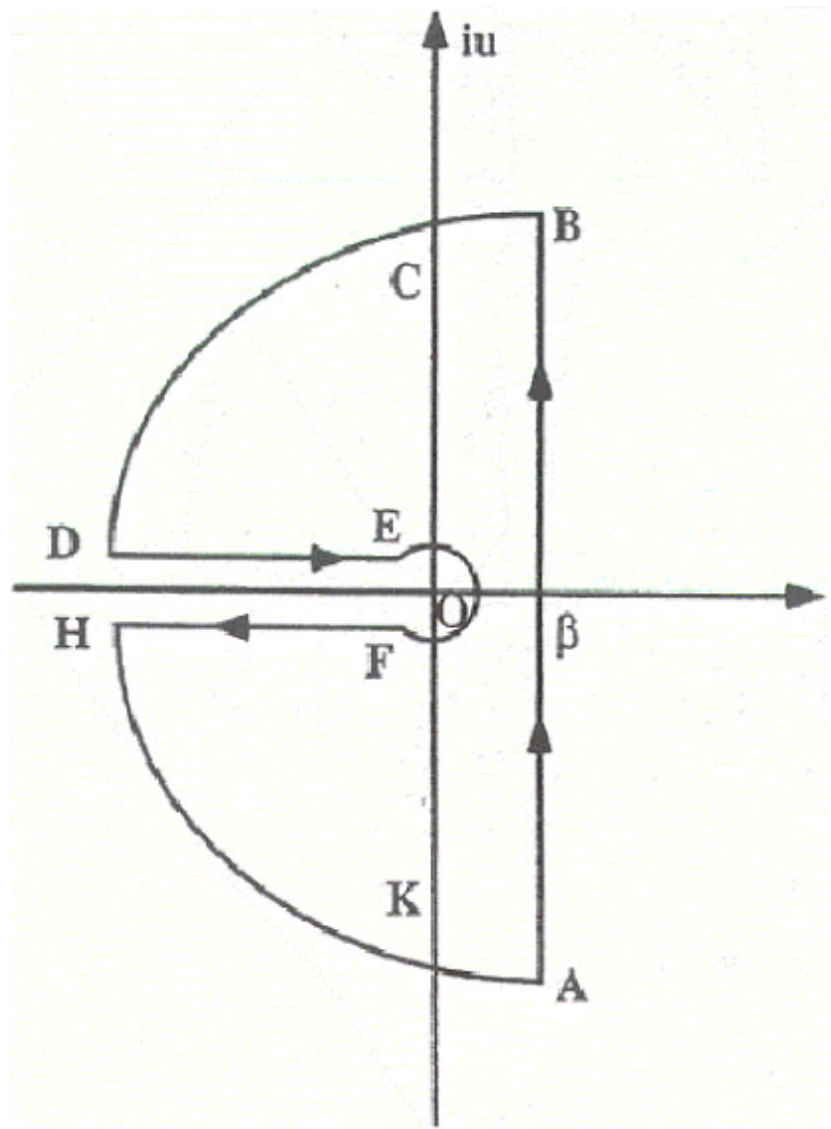

Fig. B1. Path of integration in the complex plane.

$N=2 B l \sin (\pi \nu)$

For the integral along $\Gamma_{F H}$ we set $s=r e^{-i \pi}$ and the same way we have

$I\left(r e^{-i \pi}\right)=I_{F H}(r)=-\frac{d B K}{\gamma} \frac{e^{-r t}}{e^{-i \pi v} r^{\nu}} \frac{\left(1+e^{Z^{*} r^{\nu}}\right)}{\left(e^{Z^{*} r^{\nu}}-1\right)}$

so that

$$
\begin{aligned}
T L^{-1}[Q(0, s)] & =q_{1}(0, t) \\
& =\frac{1}{2 \pi i} \int_{0}^{+\infty}\left[I_{F H}(r)-I_{D E}(r)\right] d r
\end{aligned}
$$

Renaming $\omega=\pi v$ and $Y(r)=\left[-\frac{d B K}{\gamma} \frac{e^{-r t}}{r^{v}}\right]$ we have

$$
\begin{aligned}
& q(0, t)=-\frac{d B K}{2 \pi \gamma} \int_{0}^{+\infty} \frac{e^{-r t}}{r^{\nu}} \\
& . \frac{2 \sin (\pi v)\left[e^{2 M r^{v}}-1\right]+4 \sin \left(N r^{\nu}\right) \cos (\pi \nu) e^{M r^{v}}}{e^{2 M r^{v}}+1-2 \cos \left(N r^{\nu}\right) e^{M r^{v}}} d r
\end{aligned}
$$




\section{Appendix C}

\section{Glossary}

\begin{tabular}{|c|c|c|}
\hline$\rho_{S}$ & {$\left[\mathrm{~g} \cdot \mathrm{cm}^{-3}\right]$} & $\begin{array}{l}\text { Mass of sand per unit vol- } \\
\text { ume }\end{array}$ \\
\hline$k$ & {$\left[\mathrm{~cm}^{2}\right]$} & Permeability \\
\hline$z$ & (dimensionless) & Porosity \\
\hline$q(x, t)$ & {$\left[\mathrm{g} \cdot \mathrm{s}^{-1} \cdot \mathrm{cm}^{-2}\right]$} & $\begin{array}{l}\text { Fluid mass flow rate in } \\
\text { porous medium }\end{array}$ \\
\hline$p(x, t)$ & {$\left[\mathrm{g} \cdot \mathrm{s}^{-2} \cdot \mathrm{cm}^{-1}\right]$} & Pressure of the fluid \\
\hline$\rho(x, t)$ & {$\left[\mathrm{g} \cdot \mathrm{cm}^{-3}\right]$} & $\begin{array}{l}\text { Variation of fluid mass per } \\
\text { unit volume in the porous } \\
\text { medium from the undis- } \\
\text { turbed condition }\end{array}$ \\
\hline$\rho_{F}$ & {$\left[\mathrm{~g} \cdot \mathrm{cm}^{-3}\right]$} & $\begin{array}{l}\text { Mass of fluid per unit vol- } \\
\text { ume }\end{array}$ \\
\hline & $\begin{array}{l}{\left[\mathrm{g} \cdot \mathrm{s}^{-2} \cdot \mathrm{cm}^{-1}\right]} \\
{\left[\mathrm{g} \cdot \mathrm{s}^{-1} \cdot \mathrm{cm}^{-1}\right]}\end{array}$ & $\begin{array}{l}\text { Bulk modulus of fluid } \\
\text { Viscosity of fluid }\end{array}$ \\
\hline$\frac{d \mu}{\gamma \rho_{F}}$ & {$\left[s^{n} \cdot \mathrm{cm}^{2}\right]$} & Pseudodiffusivity \\
\hline
\end{tabular}

Acknowledgements. The authors are grateful to G. Scarascia Mugnozza of the Department of Earth Science of Università "la Sapienza" in Rome for allowing to perform in his laboratory all the experiments described in this paper. Useful comments from $\mathrm{K}$. Roth and an anonymous referee improved the manuscript.

Edited by: A. Bardossy

\section{References}

Bear, J.: Dynamics of fluids in porous media, Elsevier, 1972.

Bell, M. and Nur, A.: Strength changes due to reservoir induced pore pressure and stresses and application to Lake Oroville, J. Geophys. Res., 83, 4469-4483, 1978.

Bagley, R. L. and Torvik, P. J.: On the fractional calculus model of viscoelastic behaviour, J. Rheology, 30(1), 133-155, 1986.

Caputo, M.: Linear model of dissipation whose Q is almost frequency independent, Geophys. J. R. Astr. Soc., 13, 529-539, 1967.

Caputo, M. and Plastino, W.: Rigorous time domain responses of polarizable media, Annali di Geofisica, 41, 3, 399-407, 1998.

Caputo, M.: Models of flux in porous media with memory, Water Resour. Res., 3, 693-705, 2000.

Caputo, M. and Kolari, J.: An analytical model of the Fisher equation with memory function, Alternative Perspectives on Finance and Accounting, 1, 1-16, 2001.
Caputo, M.: Economy equilibrium equations with memory, National Academy of Lincei, Italy, 2002a.

Caputo, M.: Evolutionary population growth with memory in a limited habitat, National Accademy of Lincei, 2002b.

Cesarone, F.: Modello di diffusione con memoria in membrane biologiche, Degree thesis, Advisors: Caputo, M. and Cametti, C., University of Rome "La Sapienza", 2002.

Cushman, J. H. and Moroni, M.: Statistical mechanics with 3DPTV experiments in the study of anomalous dispersion: Part I, 13(1), 75-80, Theory. Phys. Fluids, 2001.

Dewers, T. and Ortoleva, P.: Non linear dynamical aspects of deep basin hydrology: fluid compartment formation and episodic fluid release, Am. J. Sc., 294, 723-755, 1994.

Domenico, P. A. and Schwartz, F. W.: Physical and Chemical Hydrogeology, John Wiley, New York, 1997.

Elias; B. P. and Hajash Jr., A.: Changes in quartz solubility and porosity due to effective stress: An experimental investigation of pressure solution, Geology, 20, 451-454, 1992.

Indelman, P. and Abramovici, B.: Non local properties of non uniform averaged flow in heterogeneous media, Water Resour. Res., 30(12), 3385-3393, 1994.

Jacquelin, J.: Use of fractional derivatives to express the properties of energy storage phenomena in electrical networks, Laboratoires Alcatel de Marcoussis, 1984.

Körnig, H. and Müller, G.: Rheological models and interpretation of postglacial uplift, Geophys. J. R. Astr. Soc., 98, 243-253, 1989.

Le Mehaute, A. and Crépy, G.: Introduction to transfer motion in fractal media: the geometry of kinetics, Solid State Ionic, 9\&10, 17-30, 1983.

Mainardi, F.: Fractional relaxation-oscillation and fractional diffusion-wave phenomena, Chaos, Solitons and Fractals, 7, 1461-1477, 1996.

Podlubny, I.: Fractional differential equations, Academic press, 1999.

Roeloffs, E. A.: Fault stability changes induced beneath a reservoir with cyclic variations in water level, J. Geophys. Res., 93, 21072124, 1998.

Sposito, G.: General criteria for the validity of the BuckinghamDarcy flow law. Soil Sci. Soc. Am. J., 44, 1159-1168, 1980.

Steefel, C. I. and Lasaga, A. C.: A coupled model for transport of multiple chemical species and kinetic precipitation/ dissolution reactions with application to reactive flow in single phase hydrothermal system, Am. J. Sc., 294, 529-592,1994. 